BAYLEY SCORES IN LOW BIRTH WEIGHT INFANTS: FACT OR

67 FICTION. Anne Koons, Mathias Hagovsky, Alberto

Chavez, Shyan Sun (Spons. F. Behrle) UMD-New Jersey Med. Sch., Div. Neonatal-Perinatal Medicine, Newark, New Jersey Developmental follow-up studies of low birth weight infants (BSID) as an assessment tool. Implicitly or explicitly, BSID are used as predictors of subsequent outcome. Age corrections to adjust for prematurity are routinely used. We studied to adust asessments on 38 LBW infants of gestational developmental asessments on 38 LBW infants of gestational ages 25-35 weeks who had serial 6 monthly evaluations,
including BSID, and evaluated our results at postnatal age including BSID, and evaluated our results at postnatal age
(PA) after date of birth and corrected age (CA) for prematurity. Correlation analysis of test results at ages $6 \pm 2$ mo, $12 \pm 2$ mo, $18 \pm 2$ mo and $24 \pm 2$ mo against $\mathrm{I}_{Q}$ testing by Stanford Binet after age $2 \frac{1}{2}$ years was employed.

\begin{tabular}{|c|c|c|c|c|}
\hline \multirow{2}{*}{\multicolumn{2}{|c|}{$\underline{6+2 \mathrm{mo}}$}} & \\
\hline & & $12+2 \mathrm{mo}$ & $18+2 \mathrm{mo}$ & $24+2 \mathrm{mo}$ \\
\hline $\mathrm{CA}$ & $r=.63$ & $r=.41$ & $r=.64$ & $r=.76$ \\
\hline$P A$ & $r=.60$ & $r=.30$ & $r=.56$ & $r=.70$ \\
\hline & NS & NS & NS & NS \\
\hline
\end{tabular}

For the entire group there were no statistically significant advantages in employing the CA rather than PA as a predictor of later outcome. However, for the subset of infants $<32$ weeks gestation the use of $C A$ at 12 and 18 months improved the correlation with later $I Q$ testing. BSID are a limited parameter for early developmental surveillance of LBW infants and are not superior to careful pediatric evaluation.

NEURODEVELOPMENTAL OUTCOME OF INBORN 68 INFANTS $\leqslant 800$ GMS AT BIRTH. Savitri P. Kumar, Papadopoulos. Endla K. Anday, ${ }^{*}$ Rosalyn Y. Ting, ${ }^{*}$ and Maria DelivoriaNeonatology and Child Development, Phila., PA.

Seventy-eight infants $\leq 800 \mathrm{gms}$ at birth were delivered at the Hospital of the University of Pennsylvania between January 1980 and December 1982. Twenty-nine $(37 \%)$ infants survived the neonatal period. Four infants subsequently died in the hospital of sepsis and/or severe Four infants subsequently died in the hospital of simonale. Of the 25 infants discharged alive, 3 died shortly after discharge from sudden infants discharged alive, 3 died shortly after discharge from sudden infant death syndrome. - One infant was placed for adoption and moved were followed up was $690 \mathrm{gms}$ (range 475-800 gms) and 27.5 wks (range $25-30 \mathrm{wks}$ ), respectively. Eleven (52\%) of the 21 infants were small for gestational age (SGA). The oldest child is almost 4 yrs while the youngest is 1 yr of age. Rehospitalization was necessary in 10 of 21 infants for inguinal herniorrhaphy and/or lower respiratory tract infection. Growth failure was common and was noted in $52 \%$ of the fection. Growth failure was common and was noted in $52 \%$ of the fibroplasia leading to unilateral blindness was noted in 1 infant who is fibroplasia leading to unilateral blindness was noted in 1 ildly delayed (DQ 70-80, Gessell); 3 infants have developmental delay with DQ less than 70 , one of whom is microcephalic; 1 infant has mild spastic quadriplegia with DQ between 70 and 80 . None of the infants are severely handicapped, and 4 out of the 5 who developed a handicap are SGA. It appears that the outcome of inborn infants $\leq 800$ gms is very encouraging with only $24 \%$ of the population having handicaps which are mild. However, they are at greater risk for sudden infant death syndrome and growth retardation.

SLEEP PRACTICES AND SLEEP PROBLEMS IN U.S. AND JAPAN 69 S. Latz, B. Lozoff, A. Wolf, (Spon. by J. Kennell)

Most U.S. pediatricians advise parents to sleep apart from their children, primarily to promote early autonomy and avoid sleep problems. In order to understand the relationship between sleep practices and sleep problems, two highly industrialized countries with contrasting approaches to sleep management were compared. Mothers of healthy 6-' to 48-month-old children were interviewed in the U.S. and Japan. The 42 U.S. and 44 Japanese children averaged two years of age and all had been breast fed, had two-parent families and had college-educated mothers. In certain aspects of sleep management, i.e., maintaining a regular time for bed and a bedtime routine, making a firm response to bedtime struggles or night waking, and co-sleeping for part of some nights, no U.S.-Japan differences were found. However, other differences were marked ( $p$ values $<0.001$ ). Only one U.S. child's bed was in the parents' bedroom in contrast to $64 \%$ in Japan. Seventeen per cent in the U.S. had adult company and body contact when falling asleep vs. $68 \%$ in Japan. Only one U.S. child regularly slept all night with parents vs. $48 \%$ in Japan. Contrary to pediatric prediction of increased sleep problems with co-sleeping, there were no differences in night waking between the two cultures $(p=0.70)$, and there were fewer bedime conflicts in the Japanese group $(p<0.05)$. These results indicate that co-sleeping is not necessarily associated with sleep disturbances and that parental presence at bedtime may minimize conflict.
USE OF A TOUCH SENSITIVE COMPUTER TABLET FOR THE

70 ouAntitative anAlysis OF HANDWRIting IN ATTENTION DEFICIT DISORDERED (ADD) CHILDREN. MarC A. Lerner, James M. Swanson and Paul S. Lubinsky (spon. by John D. Madden). University of California, Irvine-California College of Medicine, Dept. of Pediatrics, Orange, CA

significant gaps exist in our knowledge of the basis for mild to moderate school disabilities. Children with ADD have a high incidence of dysgraphia along with their behavioral and learning difficulties. Elements which contribute to writing problems include altered neuromaturation, inattention, and fine motor inefficiency. A significant proportion of children with ADD show an improvement in written work when receiving methylphenidate (MP). The usual evaluation of handwriting problems involves a global review of the products of writing without attention to the details of the writing act. Recent computer technology offers tools for the study of important writing variables. We used a microcomputer and a touch sensitive computer tablet to quantify the writing efforts of patients who were undergoing a concurrent double blind placebo controlled assessment of cognitive and behavioral effects of MP. We obtained samples including a) the child's name; b) a spelling word; and c) a set of matched letters. A counterbalanced order of medication and placebo conditions were used to control for practice and order effects. The follow ing quantitative measures of handwriting are analyzed: speed of writing, size of letters, number of stokes per letter, pencil lifts, and fluidity. Analysis of variance indicates significant effect of MP on some of these variables. This system offers a basis for study of writing remediation programs.

\section{- 71} D. Madden) James $M$. Swanson and Paul S. Lubinsky (spon. by John Medicine, Dept of Pediatrics, Orange, CA

Behavioral problems (e.g. hyperactivity, attentional deficits, conduct disorders) have been interpreted as "masking symptoms" of depression. The recent development of the DST as a biological marker of endogenous depression in adults may be extended to children to provide an objective evaluation of masked depression. Study of the effects of dexamethasone (DEX) cose (.5 $\mathrm{mg}$ and $\mathrm{l} \mathrm{mg}$ ) and concurrent treatment with methylphenidate (MP) on the DST in ADD childxen is necessary for interpretation of results since a) dose of DEX effects sensitivity and specificity of the test, and b) common side effects of MP (e.g. anorexia and insomnia) are known to produce false positives in adults. We evaluated 50 ADD children to determine a) if age or size affected the DST; b) if, under double blind conditions, concurrent treatment with MP affected the DST; and c) to determine the sensitivity and specificity of DST in identifying children who manifest concurrent symptoms of depression. Age and size had no effect on DST results nor did use of MP. Our results confirmed the expected sensitivity (70\%) of the .5 mg DST; $20 \%$ of our subjects manifested symptoms of depression and 708 were nonsuppressors of cortisol. The
specificity of the $.5 \mathrm{mg}$ DST seems unacceptably low at first specificity of the $.5 \mathrm{mg}$ DST seems unacceptably low at first
glance, since 508 of the ADD patients without depression were also nonsuppressors, indicating endogenous depression. Either the DST is nonspecific or many ADD patients are masking symptoms of depression.

ACOUSTIC CRY FEATURES AND LATER OUTCOME IN TERM AND 72 PRETERM INFANTS. Barry M. Lester (Spon. by T. Berry Brazelton)

The objective of this study was to determine the relationship between acoustic measures of the infant cry and later mental performance in term and preterm infants. Crying was recorded at term in 20 term and 20 preterm infants. Acoustic cry features were extracted through computer analysis using the Fast Fourier Transform. Categories of cry features were derived that measure respiratory influences (duration and amplitude), neural control of the intrinsic muscles of the larynx (fundamental fre quency (FO) and variability in FO) and the configuration of the vocal tract (first and second formant frequencies or Fl and F2) The infants were tested on the Bayley scales at 18 months of age. Relationships between the acoustic cry features and the using correlation and partial correlation. The results showed significant and independent relationships between the duration (p/.05), Fo (p L.01) and variability in Fo (p/.05) of the cry
and MDI scores in term and preterm infants. Lower mental perand MDI scores in term and preterm infants. Lower mental perFo and more variability in Fo. The results show that acoustic features of the newborn cry relate to later development. Acoustic cry analysis may provide a non-invasive assessment of the nervous system that can be used in the early detection of which at-risk infants are headed for later handicap. 